\title{
Evaluation of Coherence Between ECG and PPG Derived Parameters on Heart Rate Variability and Respiration in Healthy Volunteers With/ Without Controlled Breathing
}

\author{
Hao-Yu Jan ${ }^{1} \cdot$ Mei-Fen Chen ${ }^{1} \cdot$ Tieh-Cheng Fu² $\cdot$ Wen-Chen Lin $^{1,3}$ (D) Cheng-Lun Tsai ${ }^{3,4} \cdot$ Kang-Ping Lin $^{1,3}$
}

Received: 18 October 2018 / Accepted: 23 February 2019 / Published online: 7 March 2019

(c) The Author(s) 2019

\begin{abstract}
Introduction Photoplethysmography (PPG) is used as a surrogate of electrocardiograms (ECG) for heart rate variability (HRV) analysis or respiratory rate monitoring. PPG is a more convenient way to measure HRV than ECG at rest, since respiration could be a confounding factor in HRV evaluation. However, it remains unclear whether or not controlled breathing affects breath-volume and breathing rate when HRV and pulse rate variability (PRV) are measured in different situations. Consciously controlled breathing was performed to alter the autonomic nervous states of subjects caused by respiratory sinus arrhythmia (RSA). The aim of this study was to investigate the coherence between parameters derived from ECG and PPG on healthy subjects with/without controlled breathing.

Method With 30 healthy volunteers, we measured their respiratory frequency and recorded their ECG and PPG signals during spontaneous breathing and controlled breathing, including natural paced breathing, rapid and deep breathing, slow and deep breathing, rapid and shallow breathing, and slow and shallow breathing.

Results Obvious coherence was observed between pulse rate and heart rate in both spontaneous breathing and controlled breathing tasks. However, a comparison of PRV and HRV indices demonstrated significant differences during controlled breathing. The results based on time domain and nonlinear method analysis showed that the frequency-dependent changes have more of an impact. The results also indicated that breathing corresponded well in ECG-derived parameters comparing with PPG-derived ones.

Conclusion We concluded that PPG-based devices cannot be applied as a precision screening tool to detect HRV, particularly during the cardiopulmonary analysis for the controlled breathing maneuver.
\end{abstract}

Keywords Consciously controlled breathing $\cdot$ Respiratory sinus arrhythmia $\cdot$ Pulse rate variability $\cdot$ Cardiopulmonary

$\begin{array}{ll}\text { Abbreviations } \\ \text { ECG } & \text { Electrocardiograms } \\ \text { HR } & \text { Heart rate } \\ \text { HRV } & \text { Heart rate variability } \\ \text { LF } & \text { Left index finger }\end{array}$

Wen-Chen Lin

lin_wenchen@cycu.org.tw

1 Department of Electrical Engineering, Chung Yuan Christian University, Taoyuan, Taiwan

2 Department of Physical Medicine and Rehabilitation, Chang Gung Memorial Hospital, Keelung, Taiwan

3 Technology Translation Center for Medical Device, Chung Yuan Christian University, Taoyuan, Taiwan

4 Department of Biomedical Engineering, Chung Yuan Christian University, Taoyuan, Taiwan
LT Left big toe

NPB Natural paced breathing

PPG Photoplethysmography

PPI Peak to peak interval

PR Pulse rate

PRV Pulse rate variability

RDB Rapid and deep breathing

RF Right index finger

RSA Respiratory sinus arrhythmia

RSB Rapid and shallow breathing

RT Right big toe

SDB Slow and deep breathing

SDNN Standard deviation of normal to normal intervals

SDPP Standard deviation of all peak to peak intervals

SSB Slow and shallow breathing 


\section{Introduction}

Heart rate variability (HRV) refers to the variation of beatto-beat interval, and it provides a non-invasive assessment of the balance between the cardiac sympathetic nervous system and the parasympathetic nervous system. HRV metrics has become a popular tool for diagnosis and prevention of some cardiovascular diseases such as sudden cardiac death and arrhythmia [1]. RR intervals with varying amplitudes are presented to enable the estimation of the respiratory rate and the temporal pattern of respiration [2]. Hence, electrocardiogram (ECG)-derived respiration in the context of HRV studies has been described in the literature $[3,4]$. HRV analysis and extraction of a respiratory signal from the ECG are important way to assess the heartbeat. In addition, the subtle change of vessel pulse periods is denoted as pulse rate variability (PRV). Photoplethysmography (PPG) technology has developed to include the use of small, wearable, pulse rate sensors. PPG is predominantly carried out with finger or ear lobe sensors, and occasionally from the toe or nose to noninvasively monitor the arterial oxygen saturation and pulse rates. Therefore, wearable PPG sensors have become a very popular way to measure heart rate (HR) in clinical medical practice and research proposals because of its convenience [5]. Because measuring PPG is more convenient than ECG, PPG is proposed as an alternative source, a surrogate of ECG for HRV analysis $[6,7]$ or respiratory rate monitoring [8].

Some researchers [9-12] have correlated PRV and HRV and confirmed PRV as a good surrogate of HRV, which has been debated for a decade because respiration could be a confounding factor in HRV evaluation. Respiratory sinus arrhythmia (RSA) is reflected in high-frequency HR oscillations (usually $>0.15 \mathrm{~Hz}$ in the power spectrum of R-R intervals) [13], which refers to cyclical fluctuations in HR that are coincident with the respiratory cycle, and RSA increases during inhalation and decreases during exhalation. Hence, RSA is known to have important regulatory functions, and it is the simplest and most obvious phenotype of HRV. Respiration should affect both HRV and PRV because the volume and rate of respiration could affect HR. Some studies [14-17] have focused on pulse wave research, which seeks to address whether or not the ECG-based method determining HRV can be replaced by a technique associated with pulse wave measurement. These studies suggested that respiration, a confounding factor for HRV evaluation, should be considered during consciously controlled breathing. However, little is known about the direct effect of respiration on HRV, and specifically on actual differences between PRV and HRV. In addition, there is not enough evidence to evaluate the coherence between the measurements.
Changes occur at R-R intervals, which may be affected by breathing, physical exercise, mental stress, hemodynamic alterations, or metabolic changes. Observing the change in HR during breathing manipulation (i.e. RSA) is the most direct way to present actual differences between PRV and HRV because respiration frequency and depth strongly determine fluctuation amplitude of RSA [18]. As described above, the variance of RSA is a way to understand the coherence between heart rate variability and respiration derived from ECG and PPG. Hence, a one-lead ECG signal and four channel PPG signals were used to measure RSA during controlled breathing in this study. For instance, the ECG signal was determined from Lead II, and four limbs were used to determine the PPG signals. All subjects enrolled in this study performed both breathing rate and tidal volume manipulation in order to examine the effects of breathing variations on the parameters derived from $H R$ and PR, and HRV and PRV. Thus, the aims of this study were as follows: (1) to examine whether or not the PR and PRV of either limb can be a surrogate of the HR and HRV in healthy subjects, (2) to examine whether or not the impact of HRV is equivalent to PRV during controlled breathing, and (3) to investigate cardiopulmonary coupling and its relationship with HRV during the controlled breathing tasks.

The outline of the paper was organized as follows. The procedure of gathering the signals, analysis, and their parameters were described in Sect. 2. Although HRV analysis was divided into time domain methods, frequency domain methods, time-frequency domain methods, and nonlinear methods, the short-term ( $5 \mathrm{~min})$ frequency-domain methods were not suitable for different physiological or pathological states of cardiovascular system. Hence, the most common measurements in the time domain, such as the mean of RR interval for normal beats and the standard deviation of all normal RR interval (SDNN), were used to investigate the coherence between HRV and PRV in this paper. A recurrence-plot-based analysis [19] was also employed to effectively assess the cardiopulmonary coupling of ultra-shortterm HRV signals (about $1 \mathrm{~min}$ ) during different breathing control of the subjects. The results of the comparisons were given in Sect. 3 and some concluding remarks were presented in Sect. 4.

\section{Materials and Methods}

\subsection{Subjects and Data Acquisition}

To provide a comparison between HRV derived from ECG and PRV derived from PPG, 30 healthy subjects were enrolled in this study, including 10 seniors and 20 younger individuals. Subjects, who had major cardiopulmonary disease or who were receiving regular treatments 
for diabetes mellitus, hypertension, renal or liver disease, were excluded from the study. The age rang of the younger group was 20-37 years old (mean age $=24$ years), and the senior group was 50-67 years old (mean age $=60$ years). The ratio of male and female subjects was $1: 1$ in both groups. The study was approved by the Institutional Review Board of Chang Gung Memorial Hospital, Taiwan $(101-4896 B)$. Written informed consent was obtained from all subjects enrolled in this study.

Each subject sat in a position with a comfortable posture during the experiment. Simultaneous PPG, ECG and respiratory signals were recorded using a polysomnography system (Alice 6 LDx, Philips) with a sampling frequency of $1.25 \mathrm{kHz}$ and an A/D converter (12-bit resolution). The ECG signal was determined from Lead II, the breath signal was detected via chest wall motion, and four limbs were used to determine the PPG signals, including the left index finger (LF), right index finger (RF), left big toe (LT) and right big toe (RT). Each signal was resampled to $250 \mathrm{~Hz}$ then to be analyzed by a personal computer. On account of a significant difference between HRV and PRV at higher ambient temperatures [20], all recordings were performed in a bright and quiet room with a constant temperature of $24-25^{\circ} \mathrm{C}$.

\subsection{Experimental Protocol}

In order to consider possible breathing circumstances, two different breathing circumstances were defined: spontaneous breathing and controlled breathing (Phase I and Phase II). The experimental protocol included five tasks designed to assess breathing, including Resting I, Phase I, Resting II, Phase II, and Resting III (Fig. 1a). Each breathing task had lasted for $5 \mathrm{~min}$. Subjects breathed freely in each resting task, included Resting I, II, and III. In each controlled breathing task, such as Phase I and II, subjects followed the procedure based on the study designs.

According to previous studies [21,22], breathing frequency was defined as faster breathers ( $>15$ breaths per minute) and slower breathers ( $<15$ breaths per minute). Each controlled breathing state was performed at $1 \mathrm{~min}$ interval. In the breathing control task (see Fig. 1b), we defined five types of controlled breathings, which were natural paced breathing (NPB), rapid and deep breathing (RDB), slow and deep breathing (SDB), rapid and shallow breathing (RSB), and slow and shallow breathing (SSB). In brief, subjects were asked to inhale and exhale during NPB at 15 breaths/min intervals, and inhale and exhale deeply or shallowly at 5 and 30 breaths/min intervals in the controlled breathing tasks. An example of the polysomnography signals of each measurement during slow and deep breathing are presented in Fig. 2a.

(a)

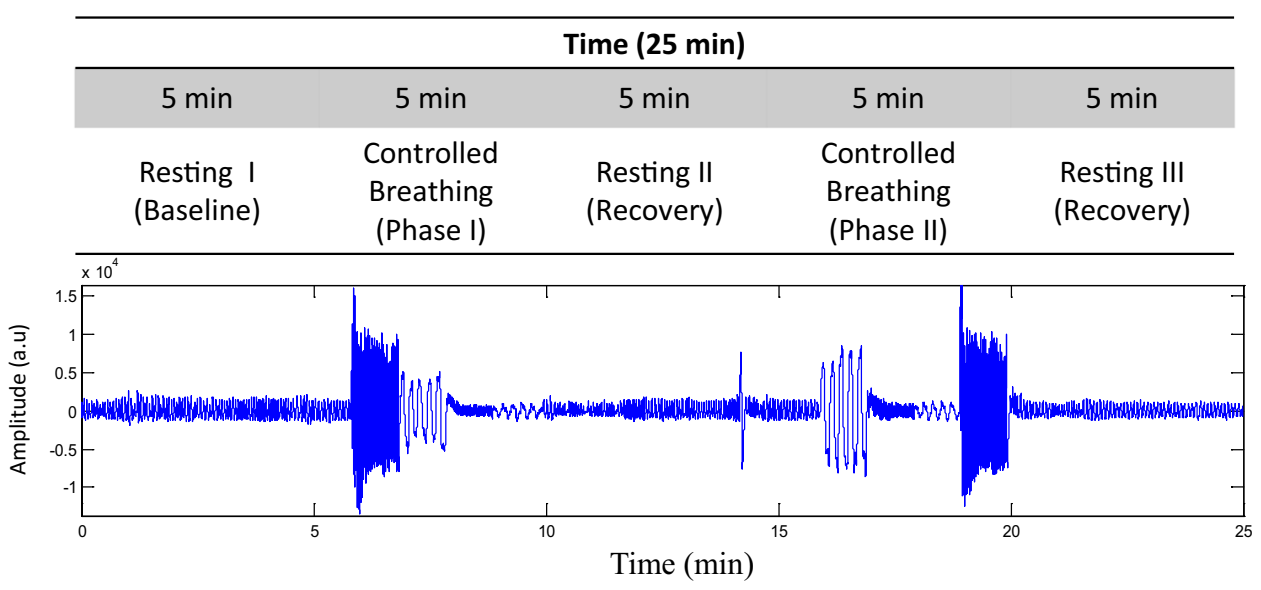

(b)

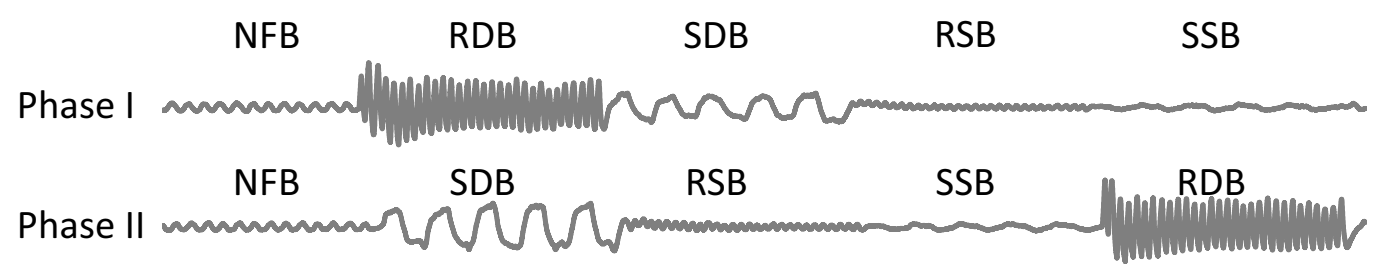

Fig. 1 a Schematic representation of experimental protocol of breathing. b Typical respiratory patterns of controlled breathing; Phase I (top) and Phase II (bottom) 


\subsection{Parameters for HRV and PRV Analysis}

HRV parameters are usually derived from $\mathrm{R}$ peak detection and generation of the $\mathrm{R}-\mathrm{R}$ interval signals.

The R-R intervals (RRI) are derived from the R peak of ECG signals. The similar procedure can be applied to PPG signals and their second derivatives to obtain P-P intervals from their upper $P$ peaks.

Representative features of ECG and PPG waves of a representative subject over a short-time interval used in HRV or PRV analysis are shown in Fig. 2b. The length of ECG signal was represented by $\mathrm{L}$, and the positions of $\mathrm{R}$ peak were determined by $\mathrm{R}$ peak detection. It was found that PPG peaks were in relation to the $\mathrm{R}$ peaks. A R peak detection algorithm utilizes double different methods [23] as the following equation:

$d_{1}(i)=x_{e c g}(i)-x_{e c g}(i-1), i=1,2, \ldots, L-1$

$d_{2}(j)=\left[d_{1}(j)-d_{1}(j-1)\right]^{2}, j=1,2, \ldots, L-2$

where $x_{\text {ecg }}(n)$ is the input signal of ECG, $d_{1}(i)$ is the first difference, and $d_{2}(j)$ is the squared double difference of the ECG signal. Then the $d_{2}(j)$ signal is filtered by bandpass filter. The dynamic threshold was determined by $80 \%$ of the peak of $d_{2}(j)$ after being filtered. In the sequence $d_{2}(j)$, the peaks of wave greater than the threshold value were selected as the windows. In the sequence $\mathrm{x}_{\text {ecg }}(\mathrm{n})$, the maxima peaks were selected within processed windows as the R-peaks. The peaks of PPG were determined by the positions between two R peaks on the ECG. RRI and PPI were defined as the follows:

$\mathrm{RRI}(\mathrm{k})=\mathrm{R}(\mathrm{k})-\mathrm{R}(\mathrm{k}-1)$

$\mathrm{PPI}(\mathrm{k})=\mathrm{P}(\mathrm{k})-\mathrm{P}(\mathrm{k}-1), \mathrm{k}=1,2, \ldots, \mathrm{N}$

Where R(i) is the R peak and P(i) is the peak of PPG. To ensure the accuracy of the detection results, all data were double-checked visually to make sure that there were no missing waves or false detection. To identify differences between HRV and PRV based on the breathing frequency and volume, some of the important time domain HRV parameters were calculated. Weinschenk et al. (2016) demonstrated some parameters via a 5-min short-term HRV with moderation to good agreements between PPG and ECG derived measurements during 1-min deep breathing tasks [24]. Therefore, based on their recommendations, appropriate parameters were used for time-domain HRV parameters. In this study, standard deviation of normal-to-normal intervals (SDNN) and standard deviation of all peak-to-peak intervals (SDPP) were the parameters to be applied.

$$
\begin{aligned}
& \text { SDNN }=\sqrt{\frac{1}{N} \sum_{i=1}^{N} \text { RRI(i) }-\overline{\text { RRI }}} \\
& \text { SDPP }=\sqrt{\frac{1}{N} \sum_{i=1}^{N} \text { PPI(i) }-\overline{\mathrm{PPI}}}
\end{aligned}
$$

Fig. 2 a A representative example of polysomnography signals using thoracoabdominal belts, PPG and ECG (from top to bottom) during deep and slow breathing. b Illustrative example of ECG and PPG waveforms. Peak detection from ECG and PPG waveform and definition of the R-R interval (RRI) and peak-to-peak interval (PPI). RRI was calculated as the time interval of two successive $R$ peaks of the ECG signal. The PPI of the upper peaks was calculated as the time interval of two successive peak of the first derivative of the PPG signal (a)

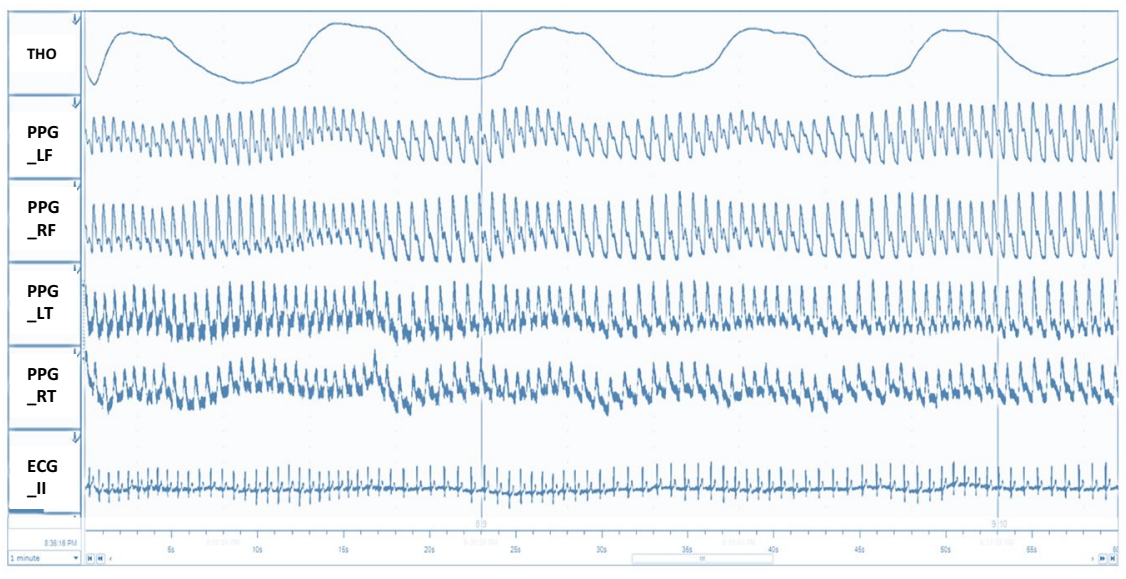

(b)

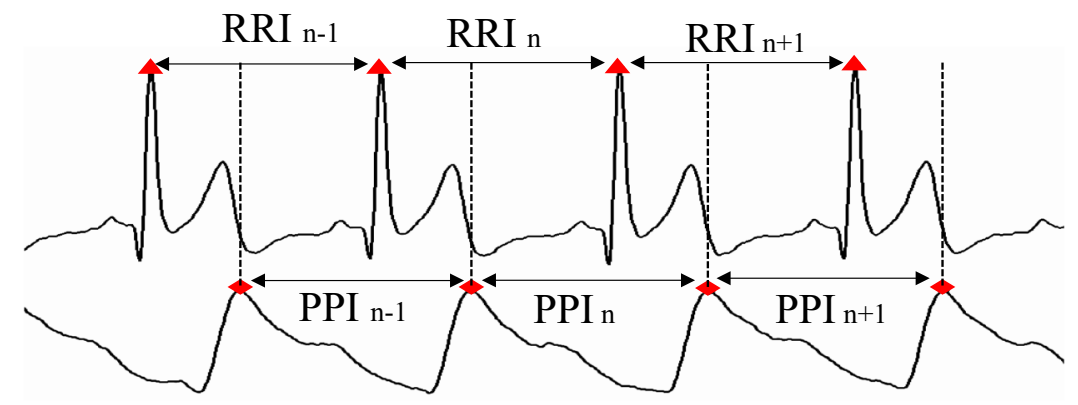


Comparisons of individual HRV and PRV were presented as the rectangular window with 5 min length.

After SDNN and SDPP were determined, the window would shift by $30 \mathrm{~s}$. Each window would yield a SDNN derived from RRI and SDPP derived from PPI.

\subsection{Coherence for Cardiopulmonary Coupling}

ECG-based cardiopulmonary coupling (CPC) is a novel HRV measure for sleep quality $[25,26]$. In order to find out the coherence between ECG-derived and PPG-derived parameters [27], a recurrence plot was used to calculate the percentage ratio between the number of recurrence points and the total number of them [28]. Figure 3 shows an example of the recurrence plot. When given two time series of $x(i)$ and $y(i)$, a recurrence plot is a representation of the normalized distance between the points (x(i), $y(i))$ and $(x(j), y(j))$, plotted in the time-to-time domain [19, 20].

The normalized distance $\mathrm{D}(\mathrm{i}, \mathrm{j})$ was calculated as follows:

$D_{(i, j)}=\sqrt{\frac{[x(i)-x(j)]^{2}}{\operatorname{var}(x)}+\frac{[y(i)-y(j)]^{2}}{\operatorname{var}(y)}}$

Where the time series $x(i)$ and $y(i)$ indicate two bidimensional cases; $\operatorname{var}(\bullet)$ indicates the time series variance. If the two points are sufficiently close to one another, i.e. the distance $D(i, j)$ is lower than a fixed cut-off value, a dot is plotted in $(i, j)$. A recurrent point in $(i, j)$ means that the interactions between the signals in the instant $i$ is almost the same as in the instant $\mathrm{j}$ (i.e. the interaction is recurring). If $D(i, j)$ is higher than the threshold [29], $(i, j)$ is not a recurrent point. A recurrent point indicates an isolated recurrence of an amplitude relationship between the signals. Percentage recurrence was defined as the percentage ratio between the number of recurrent points and the total number of all possible points. It quantified the number of time instants characterized by a recurrence in the signal interactions; the more frequent the signal dynamics, the higher the value of percent recurrence. The self-recurrence plot was applied as the baseline. To quantify the agreement, we derived the ratio of percentage recurrence from recurrence quantification analysis (RQA) according to a previous study [30]. Ratio ${ }_{R R}$ was calculated as the ratio of the range of percentage recurrence occupied by self-recurrence. Ratio ${ }_{R R}$ indicated that wherever the calculated correlation is closer to 1 , the higher the coupling of the two signals. The Ratio ${ }_{\mathrm{RR}}$ is briefly described in Fig. 4. All signals, including RRI, PPI and breathings, were normalized and resampled to $4 \mathrm{~Hz}$ to be analyzed by a personal computer. The coherence between the ECG-derived and PPG-derived respiratory signals during breathing tasks from ten subjects was analyzed to evaluate their relationships with cardiopulmonary coupling in the time domain. Figure 4 shows the coherence analysis results for one subject in the condition of NPB. As seen in Fig. 4a, a self-recurrence plot based on respiratory signals is used as the baseline. A Ratio $_{R R}$ value of $<1$ (see Fig. $4 b, c$ ) indicates that coherence was greater in the ECG-derived respiratory values than the PPG-derived ones.

\subsection{Statistical Analysis}

To compare ECG-derived parameters with PPG-derived ones, within-subject analysis during the breathing task was used. Within-subject analysis assessed the significant changes in serial results in an individual [31]. RRI and PPI were determined during spontaneous breathing and controlled breathing (Phase I). RRI and PPI among the LF, RF,

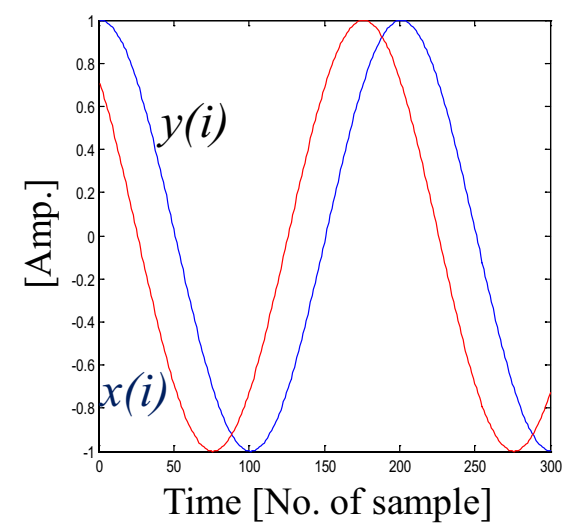

(a)

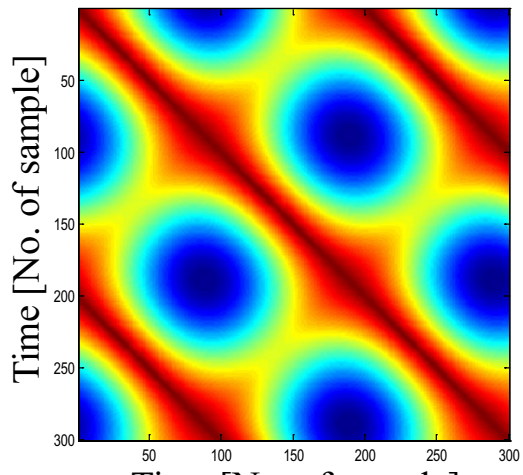

Time [No. of sample]

(b)

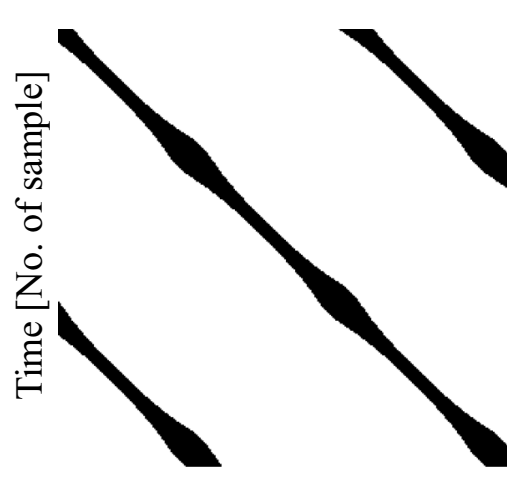

Time [No. of sample]

(c)
Fig. 3 Example of recurrence plot. Two continuous time series a, $x(i)=\sin (i)$ is sine wave and $y(i)=\sin (i+t)$ is sine wave with time delay. The normalized distance $\mathbf{b}$ was computed based on two contin- uous time series. Recurrence plot $\mathbf{c}$ was obtained by a threshold value setting form normalized distance map 


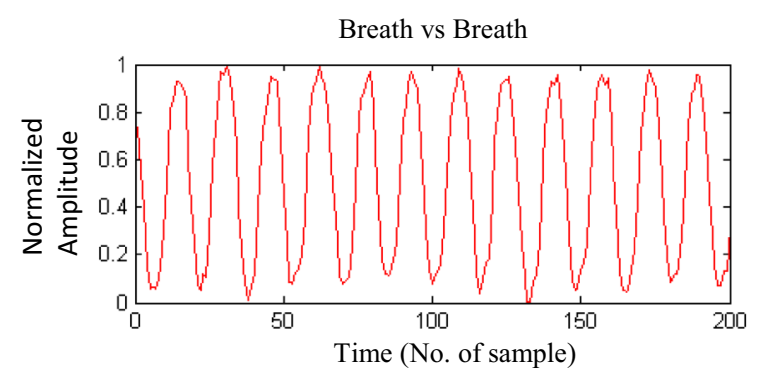

(a)

(b)

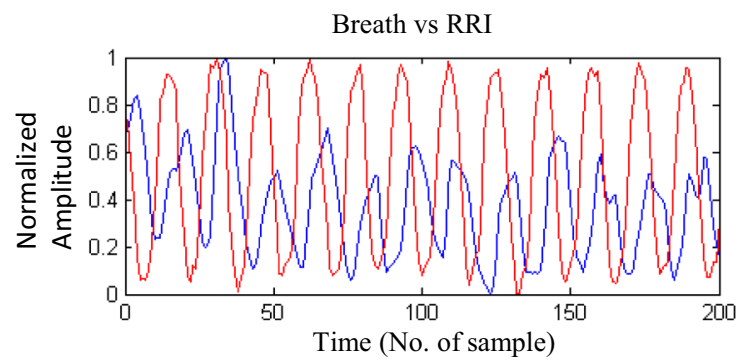

(c)

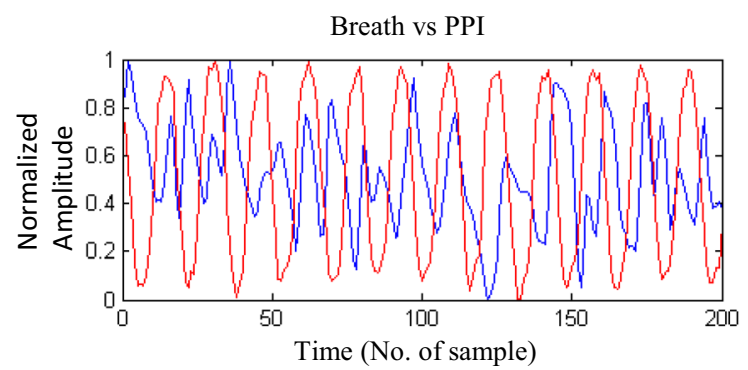

Fig. 4 Detail of the coherence analysis results at NPB for one subject: All signals, including RRI, PPI and breathing (using thoracoabdominal belts), were normalized and resampled at $4 \mathrm{~Hz}$. Illustrative example of $50 \mathrm{~s}$ sample was shown on left side. Recurrence rate was presented as the percentage of black on the recurrence plot (right side).

LT, and RT measured from each subject are compared by a Student's $t$ test, as shown in Fig. 5a. To compare HRV and PRV, a window with duration of 5 min was used. SDNN series were determined by using a window with a shift of 30 s. Each subject had 40 SDNN and SDPP. HRV and PRV were compared by the Student's t-test, as shown in Fig. 5 b. Obvious coherence was defined as a $p$-value $\leq 0.05$; insufficient coherence was indicated by a p-value greater than 0.05; Bland-Altman analysis was used to determine the $95 \%$ confidence intervals of these differences [23]. To compare HRV determined via PRV with controlled breathing, series of SDNN derived from RRI, and SDPP derived from PPI were assessed between the data from spontaneous breathing (resting baseline) and the ones during controlled breathing. Values of Ratio ${ }_{\text {RR }}$ between the two different measurements were calculated via RQA. A ratio similar to 1 was defined as coherence with respiration. Each parameter and statistical
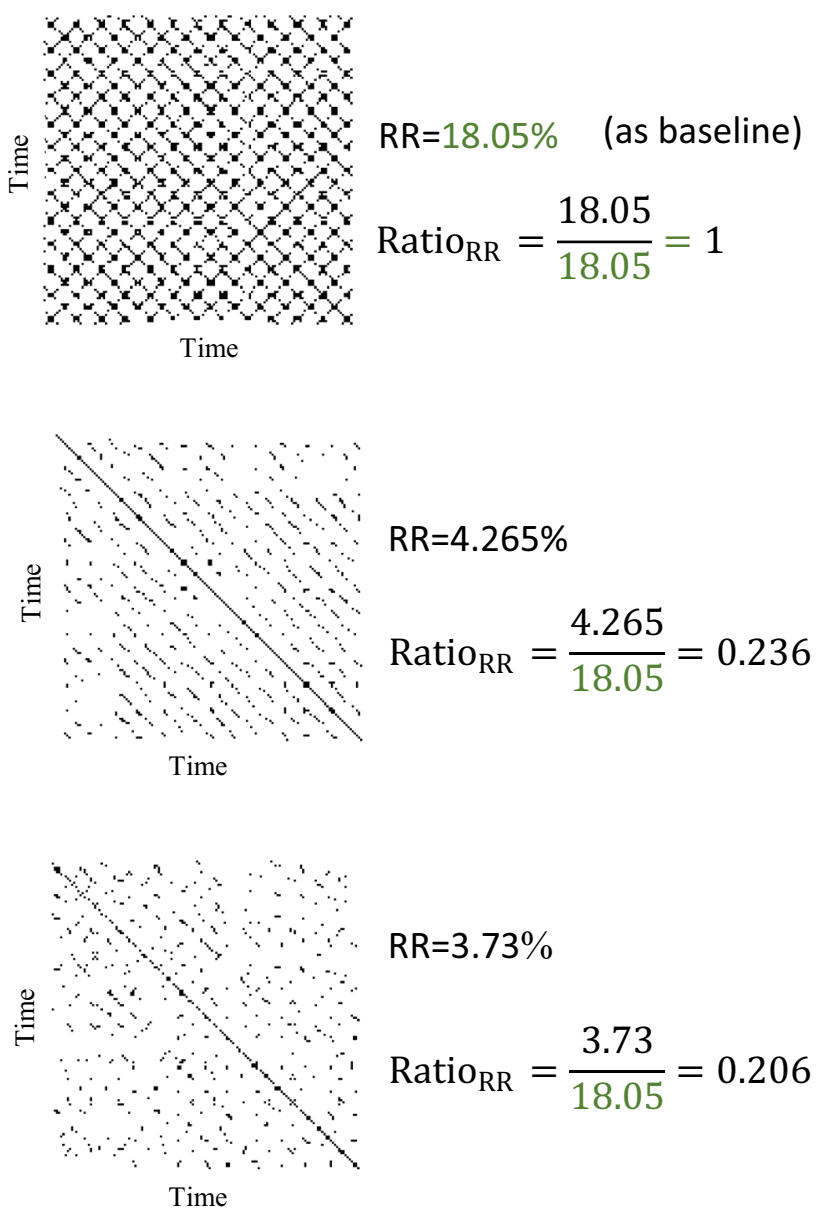

a Self-recurrence plot based on breathing signal was employed as the baseline. We found a correlation in the Ratio ${ }_{R R}$ of these recurrence plots between $\mathbf{b}$ ECG-derived respiration and $\mathbf{c}$ PPG-derived respiration

value were calculated by Mathworks MATLAB R2008a (Mathworks, Natica, MA, USA) (Table 1).

\section{Results}

\subsection{Comparisons of HR, PR/HRV and PRV}

HR and PR were compared in the breathing tasks, including spontaneous breathing and controlled breathing. For between-subjects analysis, Table 2 shows statistical comparison results of ECG-derived HR and PPG-derived PR from each limb. Good agreements and significant differences were observed between HR and PR in two types of tasks. For within-subjects analysis, Fig. 6a shows that the percentage of differences between HR and PR was almost less than $10 \%$, which demonstrated that the differences were 
(a)
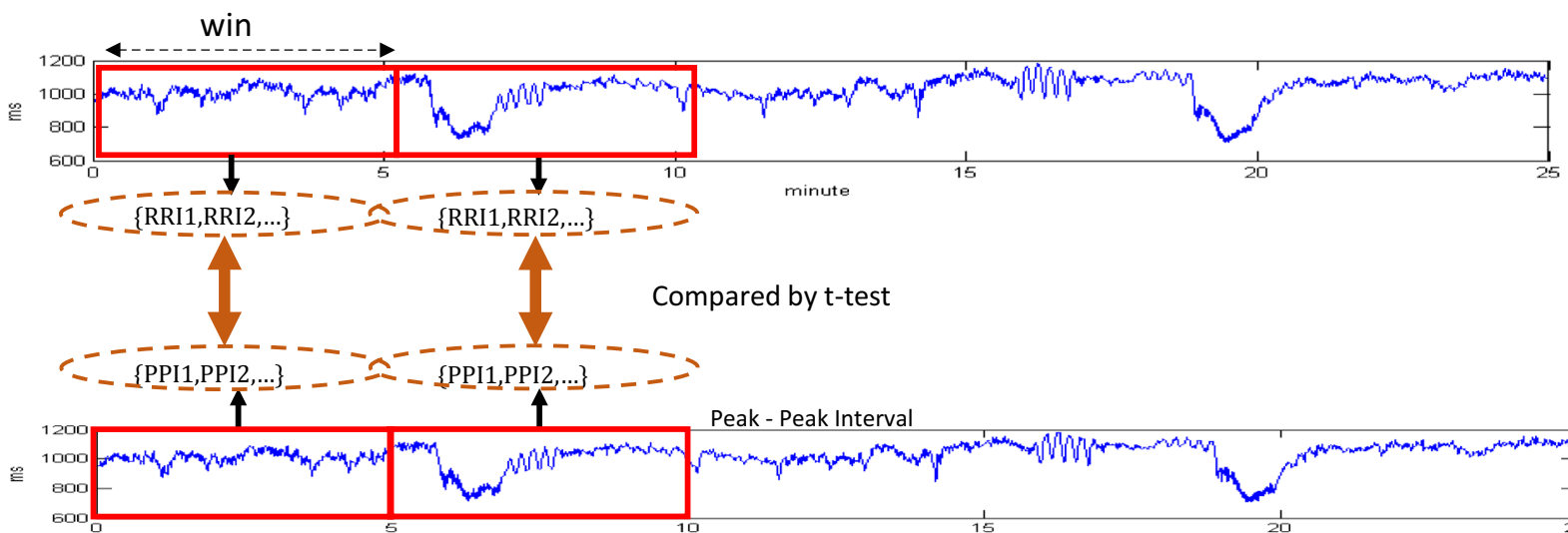

Compared by t-test

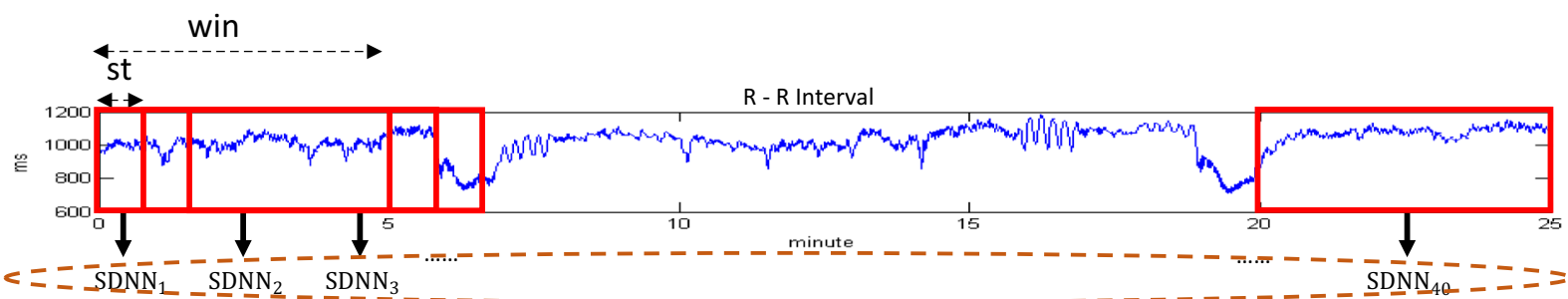

(b)

Peak - Peak Interval

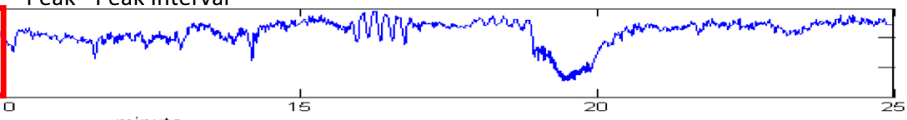

minut 
Table 2 Comparison of measurement results between ECG-derived RRI and PPG-derived PPI from each limb

\begin{tabular}{llllll}
\hline Task & RRI $(\mathrm{ms})$ & PPI $(\mathrm{ms})$ & & \\
\cline { 3 - 6 } & & LH & RH & LL & \multicolumn{2}{c}{ RL } \\
\hline Resting I (Baseline) & $872.27 \pm 144.06$ & $870.52 \pm 143.88$ & $874.13 \pm 144.73$ & $873.69 \pm 144.67$ & $874.23 \pm 143.95$ \\
& & $(0.9428)$ & $(0.0483)^{*}$ & $(0.1594)$ & $(0.0569)$ \\
Breathing (Phase & $842.74 \pm 142.87$ & $840.88 \pm 142.93$ & $841.46 \pm 143.35$ & $843.68 \pm 142.23$ & $842.50 \pm 142.86$ \\
I) & & $(0.9189)$ & $(0.9503)$ & $(0.1051)$ & $(0.6417)$ \\
Resting II (recovery) & $891.49 \pm 134.49$ & $889.07 \pm 133.62$ & $893.39 \pm 135.06$ & $893.22 \pm 135.29$ & $893.54 \pm 135.68$ \\
& & $(0.9715)$ & $(0.0608)$ & $(0.0572)$ & $(0.0084)^{*}$ \\
Breathing (Phase & $876.00 \pm 150.29$ & $876.27 \pm 150.78$ & $876.30 \pm 150.95$ & $879.62 \pm 150.02$ & $876.94 \pm 150.45$ \\
II) & & $(0.4207)$ & $(0.3066)$ & $(0.0140)^{*}$ & $(0.0461)^{*}$ \\
Resting III (recovery) & $904.00 \pm 145.44$ & $906.45 \pm 146.33$ & $906.20 \pm 145.36$ & $905.73 \pm 146.13$ & $906.76 \pm 145.78$ \\
& & $(0.0464)^{*}$ & $(0.0073)^{*}$ & $(0.0711)$ & $(0.0017)^{*}$ \\
\hline
\end{tabular}

Values from 30 healthy volunteers who were measured during spontaneous and controlled breathing are listed

RRI: R to R intervals of ECG signal; PPI: peak to peck intervals of PPG signal

Mean \pm SD ( $p$-value) bias is shown in this table. The symbol “*”means significance $(\mathrm{p}<0.05)$ between RRI and PPI form four limb. Four limbs which were refer to left hand (LH), right hand (RH), left leg (LL), right leg (RL)

\section{LF : Left Finger, RF : Right Finger , LT : Left Toe, RT : Right Toe}

\section{Spontaneous Breathing Controlled Breathing}

(a)

\begin{tabular}{|c|c|c|}
\hline & $\begin{array}{c}\text { Resting } \\
H R\end{array}$ & $\begin{array}{c}\text { Phase I } \\
\text { HR }\end{array}$ \\
\hline$P R(L F)$ & $3.33 \%(1 / 30)$ & $0 \%(0 / 30)$ \\
\hline $\mathrm{PR}(\mathrm{RF})$ & $6.67 \%(2 / 30)$ & $0 \%(0 / 30)$ \\
\hline $\mathrm{PR}(\mathrm{LT})$ & $10 \%(3 / 30)$ & $3.33 \%(1 / 30)$ \\
\hline $\mathrm{PR}(\mathrm{RT})$ & $10 \%(3 / 30)$ & $0 \%(0 / 30)$ \\
\hline
\end{tabular}

The percentage of whom have difference between $\mathrm{HR}$ and $\mathrm{PR}$

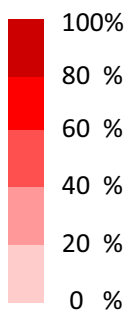

\section{Spontaneous breathing} and controlled breathing

\begin{tabular}{|c|c|c}
\hline & HRV & $\begin{array}{r}\text { The percen } \\
\text { have differ } \\
\text { HRV and PR }\end{array}$ \\
PRV(LF) & $63.33 \%(19 / 30)$ & $\begin{array}{r}100 \% \\
80 \% \\
60 \% \\
\text { PRV(RF) }\end{array}$ \\
(b) & $86.67 \%(26 / 30)$ & $40 \%$ \\
$20 \%$ \\
PRV(RT)
\end{tabular}

Fig. 6 Comparison of RRI and PPI between the spontaneous breathing and controlled breathing techniques. Within-subject analysis of the results of $\mathbf{a}$ HR and $\mathbf{b}$ HRV. LF: Left finger, RF : Right finger, LT
: Left toe, RT : Right toe. The red color bar indicates percentage difference between monitoring derived from ECG and PPG 
Table 3 Comparison of measurement results between ECG-derived SDNN and PPGderived SDPP from each limb

\begin{tabular}{llllll}
\hline Task & HRV $(\mathrm{ms})$ & \multicolumn{2}{l}{ PRV $(\mathrm{ms})$} & & \\
\cline { 3 - 6 } & & LH & RH & LL & RL \\
\hline Resting I (baseline) & $52.48 \pm 25.50$ & $54.22 \pm 23.12$ & $53.30 \pm 23.19$ & $53.92 \pm 23.77$ & $52.94 \pm 23.84$ \\
& & $(0.1253)$ & $(0.1738)$ & $(0.0660)$ & $(0.2768)$ \\
Breathing (Phase I) & $71.96 \pm 31.46$ & $73.55 \pm 29.90$ & $73.95 \pm 31.44$ & $74.44 \pm 31.55$ & $73.37 \pm 31.80$ \\
& & $(0.1524)$ & $(0.0091)^{*}$ & $(0.0002)^{*}$ & $(0.0146)^{*}$ \\
Resting II (recovery) & $55.51 \pm 25.82$ & $56.44 \pm 21.65$ & $56.06 \pm 22.94$ & $56.53 \pm 23.39$ & $55.75 \pm 22.99$ \\
& & $(0.2740)$ & $(0.3282)$ & $(0.1443)$ & $(0.4061)$ \\
Breathing (Phase II) & $72.26 \pm 30.11$ & $74.87 \pm 28.08$ & $74.44 \pm 29.93$ & $73.16 \pm 28.35$ & $73.95 \pm 30.56$ \\
& & $(0.0269)^{*}$ & $(0.0030)^{*}$ & $(0.1540)$ & $(0.0009)^{*}$ \\
Resting III (recovery) & $55.65 \pm 19.84$ & $56.24 \pm 17.62$ & $56.25 \pm 17.80$ & $56.17 \pm 18.72$ & $55.32 \pm 17.72$ \\
& & $(0.3432)$ & $(0.2939)$ & $(0.2814)$ & $(0.6454)$ \\
\hline
\end{tabular}

Values from 30 healthy volunteers who were measured during spontaneous and controlled breathing are listed

RRI: R to R intervals of ECG signal; PPI: peak to peck intervals of PPG signal

Mean \pm SD (p-value) bias is shown in this table. The symbol "*"means significance $(p<0.05)$ between SDNN and SDPP form four limb. Four limbs which were refer to left hand (LH), right hand (RH), left leg (LL), right leg (RL) and HRV indices were different significantly with breathing manipulation. Therefore, PR may be an acceptable surrogate for HR with breathing manipulation, but PRV is not an acceptable one for HRV with the manipulation.

\subsection{Impact of HRV on Breathing Manipulation}

Data from lead II of ECG were analyzed during the breathing tasks. Figure 7 shows results from the analysis of HRV and PRV via SDNN and SDPP. The differences of impact on HRV and PRV on controlled breathings between the young group and senior one were not significant. The mean SDNN of HRV is similar to that of PRV in both groups, as shown in Fig. 7a-d. The mean SDNN of HRV at SDB was 1.2-1.6 times greater than that at resting breathing. The mean SDNN of HRV at RSB was 0.5-0.7 times greater than that at resting breathing. The mean value at SDB was greater than that at the other controlled breathing tasks. The mean value of SDNN at deep breathing was greater than that at shallow breathing during rapid and slow breathing tasks. The results from PRV were similar to those from HRV. Results from the breathing tasks showed that the frequency-dependent changes on HRV have more impact than volume-dependent changes in this study. We also noted that slower breathing frequency has more impact on HRV than the rapid frequency, and the deep volume has more impact on that than the shallow volume.

\subsection{Comparison of ECG and PPG by Cardiopulmonary Coupling}

Breathing signal data were collected via chest wall motion sensor. Lead II of ECG and PPG of a left or right finger were analyzed simultaneously during the breathing tasks. The bar chart of Ratio ${ }_{R R}$ with all groups shows that the Ratio ${ }_{R R}$ of HRV is higher than that of PRV; thus, the coupling results from HRV are superior to PRV. As shown in Fig. 8a there is better breathing correspondence with ECG than PPG, particularly for the senior group (see Fig. 8b) with more differences than the young group (see Fig. 8c).

\section{Discussion}

To date, this has been the only study to assess the coherence of heart period data derived from ECG and PPG based on breathing frequency and breathing volume of respiratory analysis by within-subject analysis.

This was achieved by comparing ECG- and PPG-derived parameters by the Student's $t$-test, and the ratio of the PR range. In this study, ECG-derived $\mathrm{HR}$ and PPG-derived PR were compared in the breathing tasks, including spontaneous and controlled breathings. As expected, the results of PPGderived PR were consistent with that of ECG-derived HR recoded in the breathing interval. We found that the PPGderived PR from either limb was an acceptable surrogate of ECG-derived HR, as reported in the literature [10, 11, 24, 33]. However, no obvious coherence appeared in spontaneous breathings. This may be due to noise from the amplitude of heart rate oscillations without breathing manipulation.

The most obvious evidence was that the breath frequency $(\sim 0.2 \mathrm{~Hz})$ was predominantly detected from HRV of normal subjects during resting. Furthermore, the shifts of breathing frequency in HRV during breathing manipulation in this study also demonstrated the impact of respiration on HRV. 

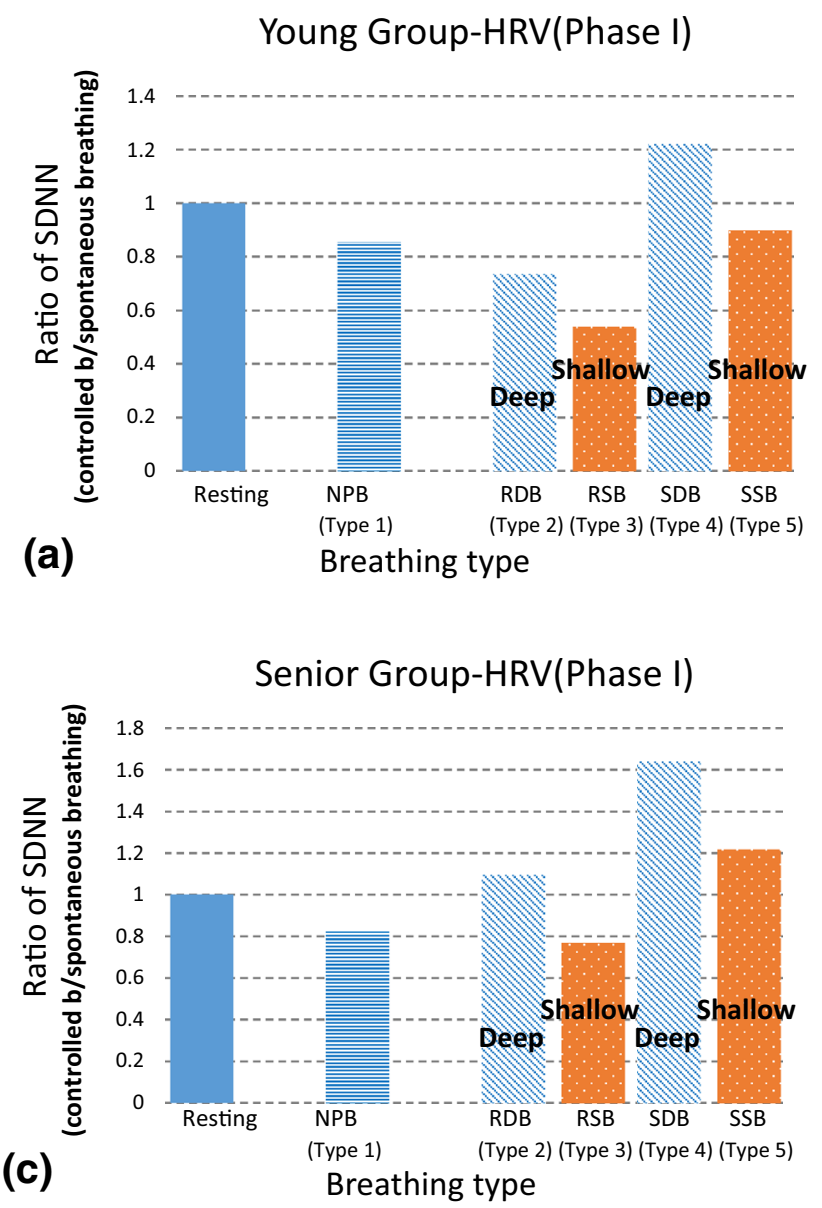

Fig. 7 Variability in heart rate amplitude during each breathing task. Values are presented as the ratio of mean of SDNN of healthy subjects at spontaneous breathing and controlled breathing (Phase I).

Hence, it is important to consider breathing state, including frequency and tidal volume, in HRV analysis and interpretation. Most studies that consider the impact of respiration on HRV only discuss either breathing frequency or tidal volume. In this study, breathing frequency and volume were discussed together. The results showed that the SDB pattern had the greatest impact on HRV and was able to change physiological parameters, such as blood pressure, which may be largely responsible for the differences [11]. When comparing our results with previous studies [10-12, 24, 32] on the impact of breathing manipulation on the normal variation of HRV, we can confirm that both SDNN and Ratio ${ }_{R R}$ in the time-domain parameters are more appropriate to analyze the impact of breathing frequency on HRV during breathing manipulation. Furthermore, an interesting finding of our study was the impact of the parameters on HRV, which was frequency $>$ volume $>$ mind, in sequence.

Breathing frequency should affect the results of HRV and PRV measures [33]. Schäfer et al. (2013) [11] investigated many studies that have explained why PPG-derived
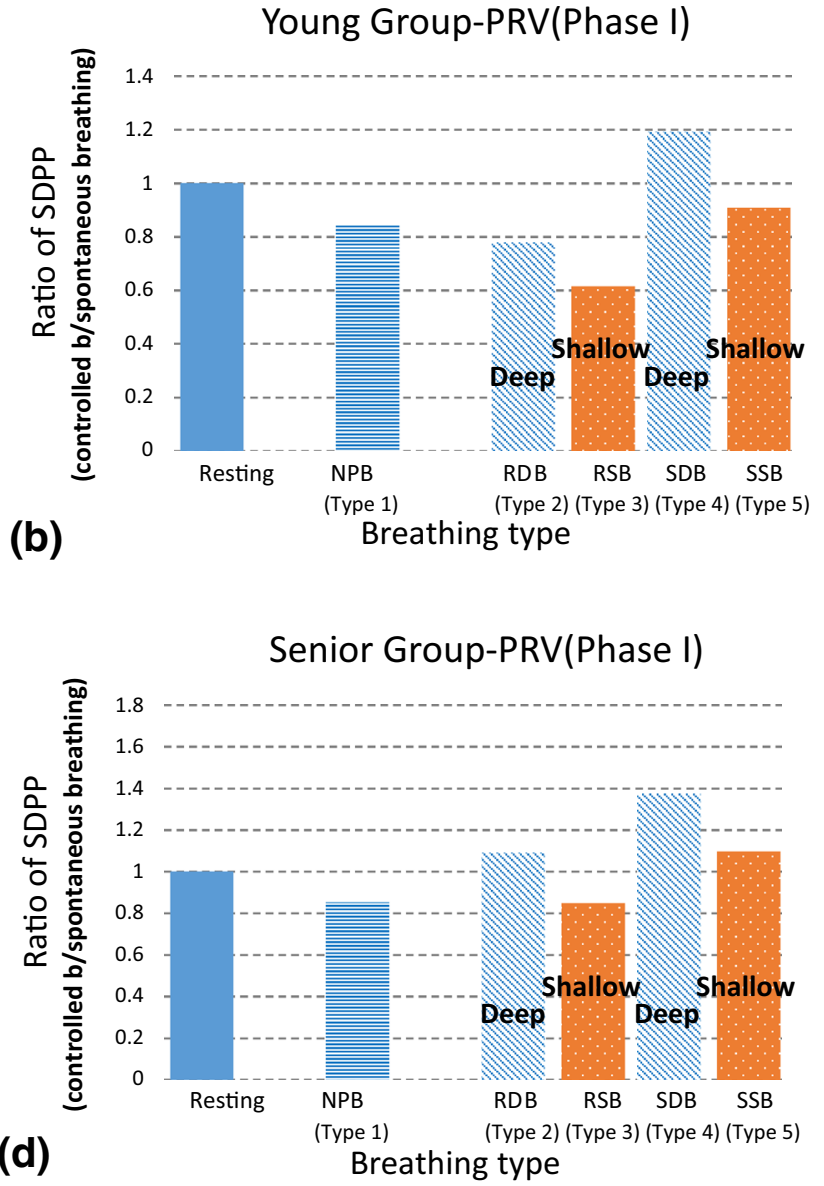

Results of a HRV and $\mathbf{b}$ PRV in the young group. Results of $\mathbf{c}$ HRV and $\mathbf{d}$ PRV in the senior group

PRV is not equivalent to ECG-derived HRV. Wong et al. (2012) [10] compared PRV variables with HRV parameters on healthy subjects and showed that PRV exhibited highly significant coherence between all HRV measures and the corresponding PRV measures of both hands. However, differences between PRV and HRV have also had been demonstrated in other studies. For example, the conclusions of a previous study [34] clearly demonstrated that respiratory pulse rate does not reflect respiratory heart rate precisely in standing subjects, and patients with low HRV. These studies have proven that physical or mental state and even body posture during PRV measurement may affect the results [35]. Hence, we analyzed heart rate variability using ECG and PPG simultaneously to assess their coherence via within-subject analysis. HRV and PRV were compared in all the breathing tasks. In addition, the fact that the differences between HRV and PRV are significant may attributable to the sensitivity of PRV parameters to pulse transients and artifacts in the breathing interval [36]. 

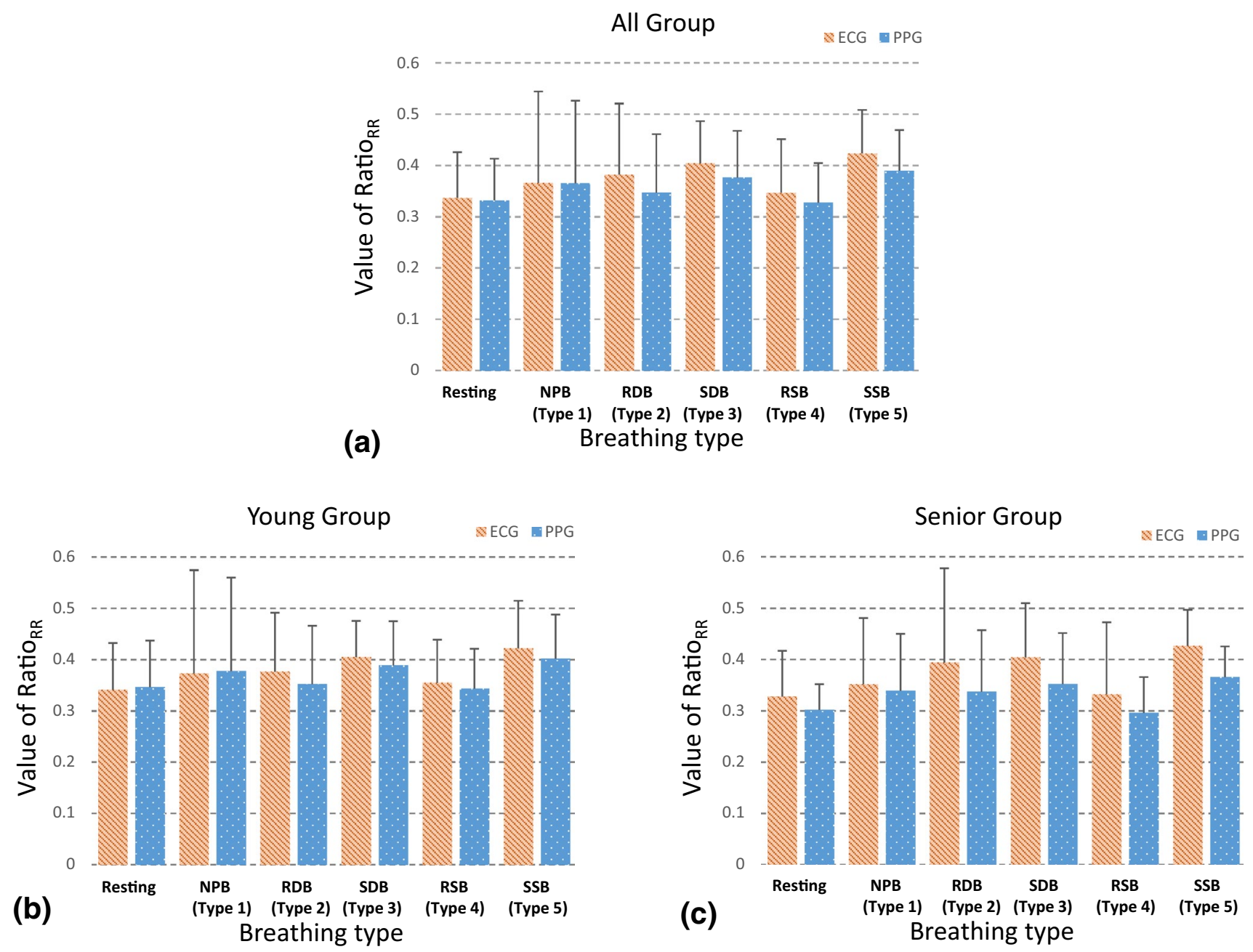

Fig. 8 Results of Ratio ${ }_{R R}$ between ECG-derived and HRV-derived parameters during each breathing task. Values of Ratio ${ }_{R R}$ were presented as mean $\pm \mathrm{SD}$ in $\mathbf{a}$ all groups, $\mathbf{b}$ the young group and $\mathbf{c}$ the senior group

Therefore, in view of this paper, the measurement results in PRV would not be replaced in HRV.

We also analyzed the coherence of cardiopulmonary coupling between PPG-derived and ECG-derived parameters to evaluate the impact of breathing manipulation. The results from all groups showed that Ratio ${ }_{R R}$ from HRV was higher than that of PRV. This indicated that the coupling result of respiratory analysis from ECG-derived HRV is superior to that of PPG-derived PRV. However, the actual physiologic meaning of HRV is not clear, particularly at very low frequency, which may suggest that the impact is associated with the surrounding soft tissue during pulse transfer. If PRV was used to replace HRV, the results of HRV will be affected by other factors to an unknown extent, as the impact of peripheral arteries on HRV remains unknown [37]. The differences in vessel status would lead to varying impact on the paths of pulse transmission. Therefore, many studies have suggested that HRV is somewhat overestimated by PRV [11].
The coherence between PRV and HRV would be impaired if subjects were undergoing physical activity or mental stress [35]; therefore, PPG-based devices are not acceptable alternatives for ECG in HRV analysis.

\section{Conclusion}

This study utilized the time-domain estimation method to investigate the coherence between HRV and respiration which parameters were both derived from ECG and PPG of healthy subjects under the circumstances of controlled breathing. Coherence analysis was used to assess the results. Respiratory fluctuations affected signals from pulse wave more than those from ECG. The velocity of the pulse wave could change because of cardiac output and varying aortic transmural pressure during mechanical breathing manipulation. Some studies have also demonstrated that respiratory 
pulse rate cannot precisely reflect respiratory heart rate in standing subjects and patients with low HRV. Therefore, we proposed that HR modulation should be considered, and ECG signals are more precise than PPG wave signals. Because of the opposing opinions, there is no conclusive consensus on whether or not the PRV of either hand can serve as a surrogate of HRV. The principal conclusion of this study is that PPG-based devices cannot be employed to detect HRV.

Acknowledgements The authors acknowledge the financial support by the Ministry of Science and Technology of Taiwan (MOST 106-2221E-033 -001- and MOST 106-2218-E-033 -009 -MY3).

\section{Compliance with Ethical Standards}

Conflict of interest The authors declare that there is no conflict of interest regarding the publication of this article.

Open Access This article is distributed under the terms of the Creative Commons Attribution 4.0 International License (http://creativeco mmons.org/licenses/by/4.0/), which permits unrestricted use, distribution, and reproduction in any medium, provided you give appropriate credit to the original author(s) and the source, provide a link to the Creative Commons license, and indicate if changes were made.

\section{References}

1. Acharya, U. R., Joseph, K. P., Kannathal, N., Lim, C. M., \& Suri, J. S. (2006). Heart rate variability: a review. Medical \& Biological Engineering \& Computing, 44(12), 1031-1051. https://doi. org/10.1007/s11517-006-0119-0.

2. Tripathi, K. K. (2004). Respiration and heart rate variability: A review with special reference to its application in aerospace medicine. Indian Journal of Aerospace Medicine, 48(12), 64-75.

3. Nemati, S., \& Malhotra, A. (2010). Clifford GD "Data fusion for improved respiration rate estimation". EURASIP Journal on Advances in Signal Processing, 2010(1), 1. https://doi. org/10.1155/2010/926305.

4. Bailón, R., \& Sornmo, L. (2006). Laguna P "A robust method for ECG-based estimation of the respiratory frequency during stress testing". IEEE Transactions on Biomedical Engineering, 53(7), 1273-1285. https://doi.org/10.1109/TBME.2006.871888.

5. Tamura, T., Maeda, Y., Sekine, M., \& Yoshida, M. (2014). Wearable photoplethysmographic sensors-past and present. Electronics, 3(2), 282-302. https://doi.org/10.3390/electronics3020282.

6. Heathers, J. A. (2013). Smartphone-enabled pulse rate variability: an alternative methodology for the collection of heart rate variability in psychophysiological research. International Journal of Psychophysiology, 89(3), 297-304. https://doi.org/10.1016/j.ijpsy cho.2013.05.017.

7. Gil, E., Orini, M., Bailon, R., Vergara, J. M., Mainardi, L., \& Laguna, P. (2010). Photoplethysmography pulse rate variability as a surrogate measurement of heart rate variability during non-stationary conditions. Physiological Measurement, 31(9), 1271-1290. https://doi.org/10.1088/0967-3334/31/9/015.

8. Nilsson, L., Johansson, A., \& Kalman, S. (2000). Monitoring of respiratory rate in postoperative care using a new photoplethysmographic technique. Journal of Clinical Monitoring and Computing, 16(4), 309-315. https://doi.org/10.1023/a:1011424732717.
9. Khandoker, A. H., Karmakar, C. K., \& Palaniswami, M. (2011). Comparison of pulse rate variability with heart rate variability during obstructive sleep apnea. Medical Engineering \& Physics, 33(2), 204-209. https://doi.org/10.1016/j.meden gphy.2010.09.020.

10. Wong, J. S., Lu, W. A., Wu, K. T., Liu, M., Chen, G. Y., \& Kuo, C. D. (2012). A comparative study of pulse rate variability and heart rate variability in healthy subjects. Journal of Clinical Monitoring and Computing, 26(2), 107-114. https://doi.org/10.1007/s1087 7-012-9340-6.

11. Schafer, A., \& Vagedes, J. (2013). How accurate is pulse rate variability as an estimate of heart rate variability?: A review on studies comparing photoplethysmographic technology with an electrocardiogram. International Journal of Cardiology, 166(1), 15-29. https://doi.org/10.1016/j.ijcard.2012.03.119.

12. Chen, X., Huang, Y. Y., Yun, F., Chen, T. J., \& Li, J. (2015). Effect of changes in sympathovagal balance on the accuracy of heart rate variability obtained from photoplethysmography. Experimental and Therapeutic Medicine, 10(6), 2311-2318. https://doi. org/10.3892/etm.2015.2784.

13. Shaffer, F., \& Ginsberg, J. P. (2017). An overview of heart rate variability metrics and norms. Front Public Health, 5, 258. https ://doi.org/10.3389/fpubh.2017.00258.

14. Kox, M., Pompe, J. C., Van Der Hoeven, J. G., Hoedemaekers, C. W., \& Pickkers, P. (2011). Influence of different breathing patterns on heart rate variability indices and reproducibility during experimental endotoxaemia in human subjects. Clinical Science, 121(5), 215-222. https://doi.org/10.1042/cs20110027.

15. Sasaki, K., \& Maruyama, R. (2014). Consciously controlled breathing decreases the high-frequency component of heart rate variability by inhibiting cardiac parasympathetic nerve activity. Tohoku Journal of Experimental Medicine, 233(3), 155-163. https ://doi.org/10.1620/tjem.233.155.

16. Lollgen, D., Müeck-Weymann, M., \& Beise, R. D. (2009). The deep breathing test: median-based expiration-inspiration difference is the measure of choice. Muscle and Nerve, 39(4), 536-544. https://doi.org/10.1002/mus.21242.

17. Saboul, D., Pialoux, V., \& Hautier, C. (2014). The breathing effect of the LF/HF ratio in the heart rate variability measurements of athletes. European Journal of Sport Science, 14, S282-S288. https ://doi.org/10.1080/17461391.2012.691116.

18. Draghici, A. E., \& Andrew Taylor, J. (2016). The physiological basis and measurement of heart rate variability in humans. Journal of Physiological Anthropology, 35, 22. https://doi. org/10.1186/s40101-016-0113-7.

19. Marwan, N., Wessel, N., et al. (2002). Recurrence-plot-based measures of complexity and their application to heart-ratevariability data. Physical Review E, 66(2), 026702. https://doi. org/10.1103/PhysRevE.66.026702.

20. Shin, H. (2015). Ambient temperature effect on pulse rate variability as an alternative to heart rate variability in young adult. Journal of Clinical Monitoring and Computing, 30(6), 939-948. https://doi.org/10.1007/s10877-015-9798-0.

21. Jennings, J. R., McKnight, J. D., \& Molen, M. (1996). Phase-sensitive interaction of cardiac and respiratory timing in humans. Psychophysiology, 33(5), 514-521. https://doi. org/10.1111/j.1469-8986.1996.tb02427.x.

22. Song, H. S., \& Lehrer, P. M. (2003). The effects of specific respiratory rates on heart rate and heart rate variability. Applied Psychophysiology and Biofeedback, 28(1), 13-23. https://doi. org/10.1023/A:1022312815649.

23. Sadhukhan, Deboleena, \& Mitra, Madhuchhanda. (2012). R-peak detection algorithm for Ecg using double difference and RR interval processing. Procedia Technology, 4, 873-877. https://doi. org/10.1016/j.protcy.2012.05.143. 
24. Weinschenk, S. W., Beise, R. D., \& Lorenz, J. (2016). Heart rate variability (HRV) in deep breathing tests and 5-min short-term recordings: agreement of ear photoplethysmography with ECG measurements, in 343 subjects. European Journal of Applied Physiology, 116(8), 1527-1535. https://doi.org/10.1007/s0042 1-016-3401-3.

25. Kesper, K., Canisius, S., Penzel, T., Ploch, T., \& Cassel, W. (2012). ECG signal analysis for the assessment of sleep-disordered breathing and sleep pattern. Medical \& Biological Engineering \& Computing, 50(2), 135-144. https://doi.org/10.1007/ s11517-011-0853-9.

26. Guo, D., Peng, C. K., Wu, H. L., Mietus, J. E., Liu, Y., Sun, R. S., et al. (2011). ECG-derived cardiopulmonary analysis of pediatric sleep-disordered breathing. Sleep Medicine, 12(4), 384-389. https ://doi.org/10.1016/j.sleep.2010.09.011.

27. Lin, Y. D., Liu, W. T., Tsai, C. C., \& Chen, W. H. (2009). Coherence analysis between respiration and PPG signal by bivariate $\mathrm{AR}$ model. World Academy of Science, Engineering and Technology, $53,847-852$.

28. Marwan, N. (2011). How to avoid potential pitfalls in recurrence plot based data analysis. International Journal of Bifurcation and Chaos, 21(4), 1003-1017. https://doi.org/10.1142/S021812741 1029008.

29. Schinkel, S., Dimigen, O., \& Marwan, N. (2008). Selection of recurrence threshold for signal detection. The European Physical Journal Special Topics, 164(1), 45-53. https://doi.org/10.1140/ epjst/e2008-00833-5.

30. Censi, F., Calcagnini, G., \& Cerutti, S. (2002). Coupling patterns between spontaneous rhythms and respiration in cardiovascular variability signals. Computer Methods and Programs in Biomedicine, 68, 37-47. https://doi.org/10.1016/S0169 -2607(01)00158-4.

31. Ricós, C., Iglesias, N., García-Lario, J. V., et al. (2007). Withinsubject biological variation in disease: Collated data and clinical consequences. Annals of Clinical Biochemistry, 44, 343-352. https://doi.org/10.1258/000456307780945633.

32. Olsson, E., Ugnell, H., Oberg, P., \& Sedin, G. (2000). Photoplethysmography for simultaneous recording of heart and respiratory rates in newborn infants. Acta Paediatrica, 89(7), 853-861. https://doi.org/10.1080/080352500750043774.

33. Schipke, J. D., Arnold, G., \& Pelzer, M. (1999). Effect of respiration rate on short-term heart rate variability. Journal of Clinical and Basic Cardiology, 2, 92-95.

34. Constant, I., Laude, D., Murat, I., et al. (1999). Pulse rate variability is not a surrogate for heart rate variability. Clinical Science, 97(4), 391-397. https://doi.org/10.1042/cs0970391.

35. Medeiros, R. F., Silva, B. M., Neves, F. J., et al. (2011). Impaired hemodynamic response to mental stress in subjects with prehypertension is improved after a single bout of maximal dynamic exercise. Clinics, 66(9), 1523-1529. https://doi.org/10.1590/ S1807-59322011000900003.

36. Hemon, M. C., \& Phillips, J. P. (2016). Comparison of foot finding methods for deriving instantaneous pulse rates from photoplethysmographic signals. Journal of Clinical Monitoring and Computing, 30(2), 157-168. https://doi.org/10.1007/s10877-015-9695-6.

37. Leicht, A. S., Crowther, R. G., \& Golledge, J. (2011). Influence of peripheral arterial disease and supervised walking on heart rate variability. Journal of Vascular Surgery, 54(5), 1352-1359. https:// doi.org/10.1016/j.jvs.2011.05.027. 